\title{
The Mechanism of Nicotinuric Acid Synthesis
}

\author{
BY K. M. JONES \\ Department of Biochemistry, University of Oxford
}

(Received 14 April 1959)

In the previous paper (Jones \& Elliott, 1959) it was shown that rat-kidney mitochondria are able to synthesize nicotinuric acid from nicotinic acid and glycine in the presence of fumarate and adenosine triphosphate (ATP). These experiments showed that nicotinuric acid synthesis is dependent upon a suitable source of energy, but did not give any other indications of the mechanism of the reaction.

Although the mechanism of nicotinuric acid synthesis has not previously been investigated, the formation of other acyl-glycine compounds, particularly hippuric acid and $p$-aminohippuric acid, has been extensively studied. The work of Chantrenne (1951) with extracts of acetone-dried ratliver powder first showed that coenzyme A (CoA) was required for hippuric acid synthesis. Subsequently, Schachter \& Taggart (1953) showed that hippuric acid synthesis proceeded in two steps, with benzoyl-CoA as an intermediate:

$$
\begin{gathered}
\text { Benzoic acid + ATP + CoA } \rightarrow \text { benzoyl-CoA } \\
+ \text { adenosine } 5^{\prime} \text {-phosphate }+ \text { inorganic pyrophosphate } \\
\text { Benzoyl-CoA + glycine } \rightarrow \text { hippuric acid + CoA. }
\end{gathered}
$$

Their enzyme preparation was able to form hippuric acid from chemically synthesized benzoyl-CoA and glycine as well as from benzoic acid, glycine, adenosine triphosphate and CoA. In a subsequent paper (Schachter \& Taggart, 1954), the specificity of the enzyme catalysing the activation of benzoic acid (reaction 1) was examined by measuring the formation of inorganic pyrophosphate when a preparation from acetone-dried ox-liver mitochondria was incubated with various acids together with adenosine triphosphate and CoA. The specificity of the enzyme catalysing reaction 2 (called glycine- $N$-acylase) for the acyl-CoA reactant was investigated by adding glycine to the incubation mixture and measuring the increase in pyrophosphate release over that brought about by reaction 1 . It was shown with butyric acid and valeric acid that the extra pyrophosphate formation caused by adding glycine was equal in amount to the butyrylglycine or valerylglycine formed. In this way both enzymes were shown to have a wide specificity among aliphatic and aromatic acids.

Among the acids tested was nicotinic acid, which gave a pyrophosphate release of $0 \cdot 13 \mu$ mole without glycine and $0.15 \mu$ mole with glycine. Thus it appears that the activating enzyme in this preparation was able to form nicotinoyl-CoA from nicotinic acid, adenosine triphosphate and CoA, but it is by no means clear from the very small increase in pyrophosphate release caused by the addition of glycine whether glycine- $N$-acylase will bring about the synthesis of nicotinuric acid from nicotinoylCoA and glycine.

In this paper it is shown that an enzyme preparation similar to that used by Schachter \& Taggart (1954) in their specificity studies can, in fact, synthesize nicotinuric acid, and its use in investigating the mechanism of this reaction is described.

\section{EXPERIMENTAL}

\section{Materials}

For the preparation and purification of materials other than those given below, see Jones \& Elliott (1959).

Nicotinoylhydroxamic acid. A solution of nicotinoylhydroxamic acid suitable for use as a chromatographic marker was prepared as follows. Hydroxylamine hydrochloride $(0.14 \mathrm{~g} . ; 2 \mathrm{~m}$-moles) and $0.1 \mathrm{~g}$. (4 m-moles) of metallic sodium were separately dissolved in the minimum volumes of dry methanol. These solutions were mixed and $0.14 \mathrm{~g}$. ( $1 \mathrm{~m}$-mole) of methyl nicotinate dissolved in $2 \mathrm{ml}$. of dry methanol was added. The mixture was left to stand at room temperature for $6 \mathrm{hr}$. and then neutralized to $\mathrm{pH} 6 \cdot 8$ with methanolic $\mathrm{HCl}$. The $\mathrm{NaCl}$ was filtered off and washed with methanol. The filtrate was made up to $20 \mathrm{ml}$. with methanol and stored at $-15^{\circ}$.

Coenzyme A. Preparations from pig liver containing $10-13 \%$ of coenzyme A (CoA) were made by the method of Elliott $(1956 a)$. Solutions were prepared in water and neutralized with KOH. A sample of $70 \%$ pure CoA was kindly supplied by Dr B. C. Loughman. CoA was assayed by the method of Kaplan \& Lipmann (1948), or that of Stadtman \& Kornberg (1953), with transacetylase from Escherichia coli, kindly prepared by $\mathrm{Dr}$ J. Lascelles. Quantities of CoA are expressed in the units of Kaplan \& Lipmann (1948).

Hydroxylamine. Hydroxylamine solutions of low salt content (salt-free hydroxylamine) were prepared by the method of Beinert et al. (1953). A saturated methanolic solution of hydroxylamine hydrochloride was neutralized to $\mathrm{pH} \mathrm{7.2}$ with strong methanolic KOH. The precipitate of $\mathrm{KCl}$ was removed by filtration and the resulting solution was evaporated to a small volume by distillation under reduced pressure. The hydroxylamine content was estimated by titration with iodine or $\mathrm{HCl}$ and the solution adjusted to $2 \mathrm{M}$ by the addition of water. This solution was stored at $-15^{\circ}$ for a maximum of 7 days and adjusted to $\mathrm{pH} 8 \cdot 0$ immediately before use. 
2-Amino-2-hydroxymethylpropane-1:3-diol buffer. This buffer (tris) was prepared by neutralizing solutions of the free base with $\mathrm{HCl}$.

\section{Methods}

Preparation of the enzyme solution. The enzyme solution used in these experiments was prepared by a modification of the method of Schachter \& Taggart (1954).

An acetone-dried powder of ox-liver mitochondria was prepared as described by Mahler, Wakil \& Bock (1953). About $30 \mathrm{~g}$. of powder was obtained $/ \mathrm{kg}$. of liver used. The acetone-dried powder was extracted by continuous stirring for $30 \mathrm{~min}$. with 10 vol. of $0.02 \mathrm{M}$-phosphate buffer, $\mathrm{pH} 7 \cdot 5$. The mixture was centrifuged and the supernatant decanted. Solid $\left(\mathrm{NH}_{4}\right)_{2} \mathrm{SO}_{4}(24 \cdot 7 \mathrm{~g}$.) was then added to each $100 \mathrm{ml}$. of the supernatant (35\% saturation). The solution was centrifuged and a further $10 \cdot 6 \mathrm{~g}$. of $\left(\mathrm{NH}_{4}\right)_{2} \mathrm{SO}_{4}$ added to each $100 \mathrm{ml}$. of the supernatant (50\% saturation). The precipitate was collected by centrifuging, made into a paste with a little water and dialysed overnight against two changes of $0.02 \mathrm{~m}-\mathrm{KHCO}_{3}$. From $64 \mathrm{~g}$. of acetone-dried powder the yield was $120 \mathrm{ml}$. of enzyme solution, which was kept at $-15^{\circ}$ for 3 months with only a small decrease in activity.

Dowex-treated enzyme. A sample of the enzyme solution was mixed with an equal volume of semi-dry Dowex-1 (10\% cross-linked) in the chloride form and stirred for $15 \mathrm{~min}$. The mixture was centrifuged and the supernatant filtered to remove particles of resin.

Measurement of activity. The standard incubation mixture used for studying nicotinuric acid synthesis had the following composition: $0.2 \mathrm{ml}$. of $0.25 \mathrm{M}$-tris buffer, $\mathrm{pH} 8.0$; $0.03 \mathrm{ml}$. of $0.1 \mathrm{M}-\mathrm{MgCl}_{2} ; 0.03 \mathrm{ml}$. of $0.1 \mathrm{M}$-adenosine triphosphate (ATP); $0.05 \mathrm{ml}$. of $0.1 \mathrm{M}$-potassium nicotinate; $0.2 \mathrm{ml}$. of $0.3 \mathrm{M}$-glycine; 40 units of $\mathrm{CoA} ; 0.25 \mathrm{ml}$. of enzyme solution; water to give a total volume of $1 \mathrm{ml}$. Incubations were performed in centrifuge tubes at $38^{\circ}$ for $30 \mathrm{~min}$. After incubation, the mixtures were deproteinized by placing in a boiling-water bath and centrifuging. Samples $(0.25 \mathrm{ml}$.) of the supernatants were taken for the estimation of nicotinuric acid by the method of Jones (1959).

The standard incubation conditions used for the investigation of nicotinoylhydroxamic acid formation were as given above except that $0.25 \mathrm{ml}$. of $2 \mathrm{M}$-hydroxylamine (salt-free) was added in place of glycine. After incubation, hydroxamic acid was estimated as described below. Nicotinoylhydroxamic acid synthesis was determined by measuring the difference in hydroxamic acid formation between incubations with and without nicotinic acid.

Estimation of hydroxamic acids. Hydroxamic acids were estimated by a modification of the method used by Kornberg \& Pricer (1953) for soluble hydroxamic acids. Incubation mixtures were deproteinized by adding $1 \mathrm{ml}$. of $7 \%$ (v/v) $\mathrm{HClO}_{4}$ and centrifuging. The supernatants were poured off into a mixture of $0.6 \mathrm{ml}$. of $7 \%(\mathrm{v} / \mathrm{v}) \mathrm{HClO}_{4}$ and $0.4 \mathrm{ml}$. of $10 \%(\mathrm{w} / \mathrm{v}) \mathrm{FeCl}_{3}, 6 \mathrm{H}_{2} \mathrm{O}$ in $0.2 \mathrm{~N}-\mathrm{HCl}$, and water was then added to bring the volume to $4 \mathrm{ml}$. Extinctions were measured at $540 \mathrm{~m} \mu$ against a reagent blank.

A standard curve for nicotinoylhydroxamic acid was obtained by the method of Elliott $(1956 a)$, modified somewhat because of differences in the methods of hydroxamic acid estimation used. Suitable volumes of a solution of methyl nicotinate in methanol $(2 \mu \mathrm{moles} / \mathrm{ml}$.) were mixed with $1 \mathrm{ml}$. of alkaline hydroxylamine solution, prepared by mixing equal volumes of $2 \mathrm{M}$-hydroxylamine hydrochloride and $2.5 \mathrm{~N}-\mathrm{NaOH}$, and methanol was added to bring the total volume to $1.5 \mathrm{ml}$. After the solutions had been set aside for $15 \mathrm{~min}$. at room temperature, $0.1 \mathrm{ml}$. of $6 \mathrm{~N}-\mathrm{HCl}$ was added to each tube, followed by $1.6 \mathrm{ml}$. of $7 \%(\mathrm{v} / \mathrm{v}) \mathrm{HClO}_{4}$, $0.4 \mathrm{ml}$. of $10 \%(\mathrm{w} / \mathrm{v}) \mathrm{FeCl}_{3}, 6 \mathrm{H}_{2} \mathrm{O}$ in $0.2 \mathrm{~N}-\mathrm{HCl}$ and $0.4 \mathrm{ml}$. of water, making a final volume of $4 \mathrm{ml}$. Extinctions were read at $540 \mathrm{~m} \mu$ against a blank solution treated as above but containing no methyl nicotinate. The extinctions obtained were proportional to the amount of methyl nicotinate added over the range $0-1 \mu$ mole.

Chromatographic methods. The chromatographic separation of tertiary nicotinic acid compounds and their detection by treatment with cyanogen bromide and $p$-aminobenzoic acid have been described by Jones \& Elliott (1959).

On chromatography in butan-1-ol-water synthetic nicotinoylhydroxamic acid gave a somewhat elongated spot with an $R_{F}$ value slightly less than that of nicotinamide. On treatment with cyanogen bromide and $p$-aminobenzoic acid it gave a yellow with a slight brownish tinge immediately on spraying, turning to canary yellow when dry; these colours allowed the nicotinoylhydroxamic acid spot to be clearly distinguished from that of nicotinamide. It also gave the brownish-purple characteristic of hydroxamic acids when sprayed with $10 \%(\mathrm{w} / \mathrm{v}) \mathrm{FeCl}_{3}, 6 \mathrm{H}_{2} \mathrm{O}$ in $0.2 \mathrm{~N}-\mathrm{HCl}$.

\section{RESULTS}

Synthesis of nicotinuric acid by the enzyme preparation. The enzyme preparation synthesized nicotinuric acid when incubated with nicotinic acid, glycine, ATP and CoA (Table 1). Nicotinic acid, glycine and ATP were all absolutely required for the synthesis. Added CoA, however, was not necessary for nicotinuric acid formation, but when $\mathbf{4 0}$ units of CoA were added to the incubation mixture the synthesis was $50 \%$ greater than in its absence.

The time course of nicotinuric acid formation is shown in Fig. 1. The synthesis proceeded linearly for $30 \mathrm{~min}$. and then fell off abruptly.

The enzyme preparation was unable to form nicotinamide from nicotinic acid when glycine was replaced by $\mathrm{NH}_{4} \mathrm{Cl}$ (3-30 mM, final concentration). This confirms results obtained with whole ratkidney mitochondria (Jones \& Elliott, 1959), indicating that nicotinamide is not synthesized by a route similar to that for nicotinuric acid formation.

\section{Table 1. Nicotinuric acid synthesis by the enzyme preparation}

Incubation conditions are given in the text.

$\begin{array}{cc}\begin{array}{c}\text { Composition of incubation } \\ \text { mixture }\end{array} & \begin{array}{c}\text { Nicotinuric acid formed } \\ (\mu \text { mole } / 30 \text { min. })\end{array} \\ \text { Complete mixture } & 1 \cdot 13 \\ \text { Nicotinic acid omitted } & 0 \cdot 0 \\ \text { Glycine omitted } & 0 \cdot 01 \\ \text { ATP omitted } & 0 \cdot 0 \\ \text { CoA omitted } & 0.75\end{array}$


Hydroxamic acid formation by the enzyme preparation. When glycine was replaced in the incubation mixture by salt-free hydroxylamine solution, the enzyme preparation formed a hydroxamic acid (Table 2). The values in Table 2 have been corrected for the hydroxamic acid-reacting material present in an incubation mixture stopped at zero time. Hydroxamic acid formation was completely dependent only upon ATP and hydroxylamine. In the absence of nicotinic acid a small hydroxamic acid blank was obtained, presumably due to traces of carboxylic acids in the enzyme solution or the CoA preparation. On the addition of nicotinic acid a large increase in hydroxamic acid formation occurred and it was assumed that only this increase represented nicotinoylhydroxamic acid. The identification of this substance with nicotinoylhydroxamic acid is described below. Nicotinoylhydroxamic acid formation occurred without added CoA, but a $47 \%$ stimulation of synthesis was observed when 40 units of CoA were added. Glutathione inhibited nicotinoylhydroxamic acid formation, $23 \%$ inhibition being found with $0.02 \mathrm{M}$-glutathione.

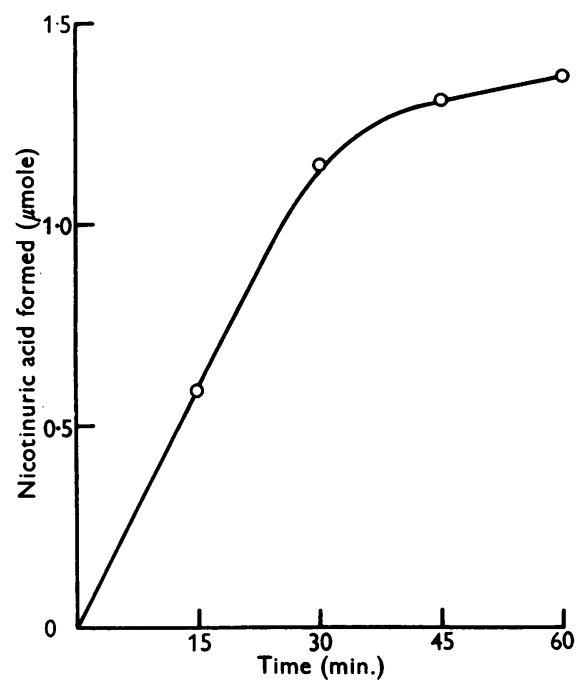

Fig. 1. Time course of nicotinuric acid synthesis. Incubation conditions are given in the text.

Table 2. Hydroxamic acid synthesis by the enzyme preparation

Incubation conditions are given in the text. The results are expressed in terms of nicotinoylhydroxamic acid and have been corrected for the zero time blank.

Composition of incubation mixture

Hydroxamic acid formed

Complete mixture ( $\mu$ moles/30 min.)

Nicotinic acid omitted

Hydroxylamine omitted

0.56

0.05

$0 \cdot 0$

ATP omitted

0.01

CoA omitted
When glycine and hydroxylamine were present in the same incubation mixture, the syntheses of both nicotinuric acid and nicotinoylhydroxamic acid were inhibited by $50 \%$ compared with the amounts obtained with glycine or hydroxylamine singly.

The effect of hydroxylamine concentration on nicotinoylhydroxamic acid synthesis is shown in Fig. 2. Maximum synthesis was obtained with a hydroxylamine concentration of $0.5 \mathrm{M}$. At $0.75 \mathrm{M} \mathrm{a}$

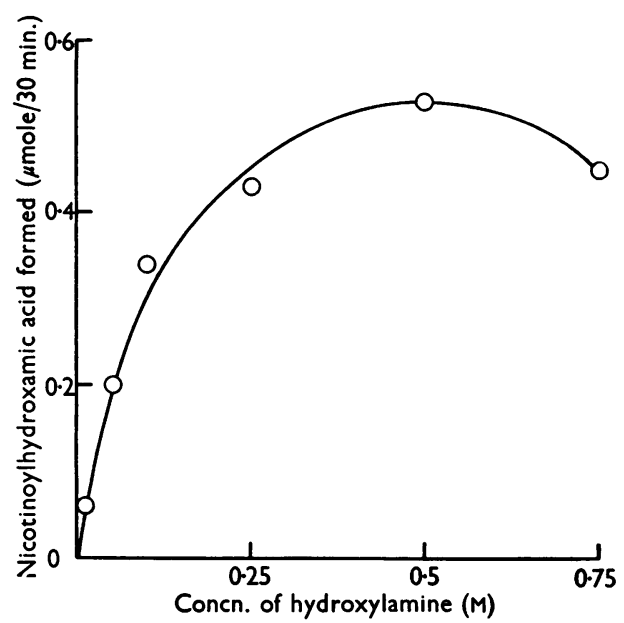

Fig. 2. Effect of hydroxylamine concentration upon nicotinoylhydroxamic acid synthesis. Values have been corrected for blanks obtained at each hydroxylamine concentration in the absence of nicotinic acid. Incubation conditions are given in the text.

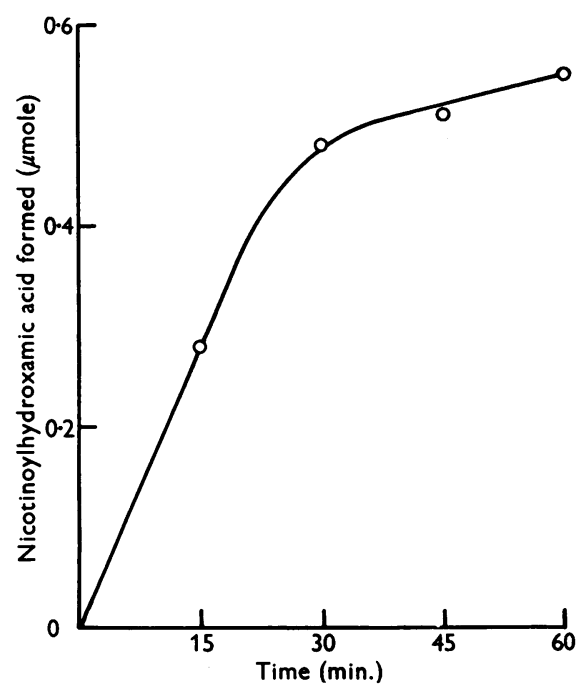

Fig. 3. Time course of nicotinoylhydroxamic acid synthesis. Values have been corrected for blanks obtained at each time in the absence of nicotinic acid. Incubation conditions are given in the text. 
small degree of inhibition was observed which may have been due to methanol present in the hydroxylamine solution.

The time course of nicotinoylhydroxamic acid formation was similar to that for nicotinuric acid formation, the rate of synthesis being constant up to about $30 \mathrm{~min}$. and then falling off (Fig. 3).

Identification of the hydroxamic acid formed by the enzyme preparation. In order to identify the enzymically formed hydroxamic acid, mixtures were set up with and without nicotinic acid and, after incubation for $30 \mathrm{~min}$. at $38^{\circ}$, the reaction was stopped by placing the tubes in a boiling-water bath for $45 \mathrm{sec}$. The mixtures were centrifuged and $0.5 \mathrm{ml}$. samples of the supernatants together with suitable markers were spotted onto Whatman no. 3 paper. Two such chromatograms were developed in butan-1-ol-water for $12 \mathrm{hr}$.

One of these chromatograms was sprayed with $10 \%(\mathrm{w} / \mathrm{v}) \mathrm{FeCl}_{3}, 6 \mathrm{H}_{2} \mathrm{O}$ in $0 \cdot 2 \mathrm{~N}-\mathrm{HCl}$ to detect hydroxamic acids. No hydroxamic acid was found in the incubation mixture without nicotinic acid. In the complete mixture a single hydroxamic acid spot having the same $R_{F}$ as synthetic nicotinoylhydroxamic acid was present, and this was reinforced when synthetic nicotinoylhydroxamic acid was added to the complete mixture after incubation.

The duplicate chromatogram was treated with cyanogen bromide and $p$-aminobenzoic acid to show up nicotinic acid derivatives. In the complete incubation mixture a spot appeared having the same $R_{F}$ as the enzymically synthesized hydroxamic acid, showing that this hydroxamic acid was also a nicotinic acid derivative. This spot corresponded, both in position and in the colours given, with synthetic nicotinoylhydroxamic acid, and was reinforced by the synthetic substance. None of the substance was present in the incubation mixture lacking nicotinic acid.

These experiments establish the identity of the enzymically-formed hydroxamic acid with nicotinoylhydroxamic acid.

Effect of coenzyme $A$. The presence of added CoA was not necessary for the enzyme preparation to form either nicotinuric acid or nicotinoylhydroxamic acid, but in both cases a stimulation of synthesis of about $50 \%$ occurred when it was added at a concentration of $40 \mathrm{units} / \mathrm{ml}$. The effect of various CoA concentrations upon nicotinoylhydroxamic acid synthesis is shown in Table 3; optimum synthesis took place with 30-40 units of CoA added $/ \mathrm{ml}$. At higher concentrations CoA inhibited synthesis, so that at 80 units $/ \mathrm{ml}$. the activity was only $7 \%$ higher than that obtained without added CoA. It is not known whether this inhibition was due to CoA itself or to other substances present in the CoA preparations, which contained only $10-13 \%$ of CoA. The effect of added CoA upon nicotinuric acid synthesis is exactly similar to its effect upon nicotinoylhydroxamic acid formation (Table 3).

It seemed from these results that $\mathrm{CoA}$ was required for the synthesis of both nicotinuric acid and nicotinoylhydroxamic acid, but that the enzyme preparation contained sufficient of the coenzyme to give considerable activity without any being added to the incubation mixtures. In order to determine more clearly whether CoA was involved in the reactions, the enzyme preparation was treated with Dowex-1 to remove CoA.

The Dowex-treated enzyme formed a small amount of nicotinoylhydroxamic acid in the absence of added CoA, but only about one-fifth of the amount formed by the untreated enzyme (Table 3). On the addition of CoA (40 units/ml.) there was a $260 \%$ increase in nicotinoylhydroxamic acid formation. The inhibition at higher CoA concentrations was still found, the activity at 80 units/ ml. showing only a $150 \%$ increase over that in the absence of CoA, and the Dowex treatment had not greatly affected the optimum concentration of CoA. The synthesis of nicotinuric acid by the Dowextreated enzyme in the absence of added CoA was likewise much lower than that by the untreated enzyme (Table 3). The addition of 40 units of CoA/ ml. caused a $270 \%$ increase in nicotinuric acid synthesis, whereas at $80 \mathrm{units} / \mathrm{ml}$. the increase observed was only $164 \%$.

In order to show that the active component in the impure (10-13\%) CoA preparations used was, in

Table 3. Effect of coenzyme $A$ on nicotinoylhydroxamic acid synthesis and nicotinuric acid synthesis

Incubation conditions are given in the text.

\begin{tabular}{|c|c|c|c|c|}
\hline \multirow[b]{2}{*}{$\begin{array}{l}\text { Conen. of CoA } \\
\text { (units/ml.) }\end{array}$} & \multicolumn{2}{|c|}{$\begin{array}{l}\text { Nicotinoylhydroxamic acid } \\
\text { formed ( } \mu \text { mole/30 min.) }\end{array}$} & \multicolumn{2}{|c|}{$\begin{array}{l}\text { Nicotinuric acid formed } \\
(\mu \text { mole/30 min. })\end{array}$} \\
\hline & $\begin{array}{l}\text { Untreated } \\
\text { enzyme }\end{array}$ & $\begin{array}{l}\text { Dowex-treated } \\
\text { enzyme }\end{array}$ & $\begin{array}{l}\text { Untreated } \\
\text { enzyme }\end{array}$ & $\begin{array}{l}\text { Dowex-treatec } \\
\text { enzyme }\end{array}$ \\
\hline 0 & 0.37 & 0.08 & 0.75 & $0 \cdot 25$ \\
\hline 20 & 0.51 & $0 \cdot 24$ & - & - \\
\hline 40 & 0.53 & $0 \cdot 29$ & $1 \cdot 13$ & 0.92 \\
\hline 60 & $0 \cdot 44$ & $0 \cdot 26$ & - & - \\
\hline 80 & $0 \cdot 4()$ & $0 \cdot 20$ & $0 \cdot 88$ & $0 \cdot 66$ \\
\hline
\end{tabular}


fact, CoA, a more highly purified preparation (about $70 \%$ of CoA) was employed. This preparation had the same stimulating effect upon nicotinoylhydroxamic acid synthesis by the Dowex-treated enzyme as the less pure preparation.

Accumulation of an intermediate in nicotinuric acid synthesis. When the Dowex-treated enzyme was incubated with nicotinic acid, ATP and CoA, but without glycine and hydroxylamine, a substance accumulated which reacted non-enzymically with hydroxylamine to form a hydroxamic acid. When glycine was present in the incubations, none of this substance was found. The formation of the substance was estimated as follows. After incubation, the mixtures were adjusted to $\mathrm{pH} 5 \cdot 0$, and deproteinized by placing the tubes in a boiling. water bath and then centrifuging. The supernatants were neutralized to $\mathrm{pH} 7 \cdot 0$ and $1 \mathrm{ml}$. of $2 \mathrm{M}$ hydroxylamine (salt-free) was added to each. The solutions were left to stand at room temperature for $15 \mathrm{~min}$. and their hydroxamic acid contents estimated. Blank values obtained with incubation mixtures containing no nicotinic acid were subtracted from the experimental values. In this way the accumulation of $0.09 \mu$ mole of the substance was shown in the incubation performed in the absence of glycine, but none was found when glycine was present in the incubations.

\section{DISCUSSION}

The high concentration of hydroxylamine required for maximal nicotinoylhydroxamic acid formation by the enzyme preparation suggests that the hydroxamic acid was formed by a non-enzymic reaction between hydroxylamine and an enzymically formed carboxyl-activated form of nicotinic acid. Since ATP and CoA were both required for the formation of nicotinoylhydroxamic acid, it seems that the activated form of nicotinic acid is nicotinoyl-CoA, and that the overall reaction proceeds in two steps:

$$
\begin{aligned}
& \text { Nicotinic acid }+ \text { ATP }+ \text { CoA } \rightarrow \text { Nicotinoyl-CoA } \\
& \text { Nicotinoyl-CoA }+ \text { hydroxylamine } \rightarrow \\
& \text { Nicotinoylhydroxamic acid }+ \text { CoA. }
\end{aligned}
$$

The results presented also show that nicotinoyl$\mathrm{CoA}$ is an intermediate in nicotinuric acid synthesis, reaction 3 being followed in this case by reaction 5 :

Nicotinoyl-CoA + glycine $\rightarrow$ Nicotinuric acid + CoA.

The nature of the ATP breakdown accompanying nicotinoyl-CoA formation has not been investigated, but it seems likely that it is the same as has been found with other carboxylic acid-activating reactions of this type, the products being adenosine $5^{\prime}$-phosphate and inorganic pyrophosphate (Lipmann, Jones, Black \& Flynn, 1952; Kornberg \& Pricer, 1953; Mahler et al. 1953; Elliott, 1957).
The mechanism proposed for nicotinuric acid synthesis is the same as that found for hippuric acid synthesis (Schachter \& Taggart, 1953, 1954). This type of mechanism for the formation of $-\mathrm{CO} \cdot \mathrm{NH}-$ bonds, involving condensation of an acyl-CoA compound with an amino group, is widespread though by no means universal, and is also found, for example, in the acetylation of aromatic amines (Stadtman, Novelli \& Lipmann, 1951; Lynen, Reichert \& Rueff, 1951) and in taurocholic acid synthesis (Elliott, 1956a, b). It seems probable that, as has been proved in the acetylation reactions and in hippuric acid synthesis, the two reactions of nicotinuric acid synthesis are catalysed by separate enzymes, though evidence for this can only be obtained by further purification of the system.

Assuming that two enzymes are involved in nicotinuric acid formation, it is uncertain whether they are the same as those synthesizing hippuric acid. The enzyme preparation used in the present experiments was very similar to that employed by Schachter \& Taggart (1954) in studies of hippuric acid synthesis, and it was shown chromatographically to be able to form hippuric acid from benzoic acid and glycine. However, it was relatively little purified from the crude extract, and it is quite possible that two or more enzymes catalysing the same type of reaction but having different specificities were present. The same considerations apply to any attempt to identify the enzyme catalysing the activation of nicotinic acid with any of the known enzymes bringing about acyl-CoA formation.

\section{SUMMARY}

1. An enzyme preparation has been obtained from acetone-dried ox-liver mitochondria which synthesized nicotinuric acid from nicotinic acid, glycine, adenosine triphosphate and coenzyme $\mathbf{A}$. It also formed nicotinoylhydroxamic acid, which was identified chromatographically, when glycine was replaced by high concentrations of hydroxylamine.

2. Both reactions occurred without added coenzyme $A$ but showed about $50 \%$ stimulation in the presence of coenzyme A (40 units $/ \mathrm{ml}$.). With a Dowex-1 treated enzyme solution, the syntheses in the absence of added coenzyme $\mathbf{A}$ were decreased several-fold, and in both cases a 260-270\% increase in activity was found when coenzyme A (40 units/ ml.) was added.

3. The mechanisms of these reactions are discussed.

I wish to thank Professor Sir Hans A. Krebs, F.R.S., and Dr W. H. Elliott for their advice and interest in this work. I am indebted to the Christopher Welch Trust, to St John's College, Oxford, and to the Medical Research Council for the 
award of Scholarships held during the course of this work. The work was aided by a grant to the Department from the Rockefeller Roundation.

\section{REFERENCES}

Beinert, H., Green, D. E., Hele, P., Hift, H., von Korff, R. W. \& Ramakrishnan, C. V. (1953). J. biol. Chem. 203, 35.

Chantrenne, H. (1951). J. biol. Chem. 189, 227.

Elliott, W. H. (1956a). Biochem. J. 62, 427.

Elliott, W. H. (1956b). Biochem. J. 62, 433.

Elliott, W. H. (1957). Biochem. J. 65, 315.

Jones, K. M. (1959). Biochem. J. 73, 719.

Jones, K. M. \& Elliott, W. H. (1959). Biochem. J. 73, 706.

Kaplan, N. O. \& Lipmann, F. (1948). J. biol. Chem. 174, 37.
Kornberg, A. \& Pricer, W. E., jun. (1953). J. biol.Chem. 204, 329.

Lipmann, F., Jones, M. E., Black, S. \& Flynn, R. M. (1952). J. Amer. chem. Soc. 74, 2384.

Lynen, F., Reichert, E. \& Rueff, L. (1951). Liebigs Ann. 574, 1.

Mahler, H. R., Wakil, S. J. \& Bock, R. M. (1953). J. biol. Chem. 204, 453.

Schachter, D. \& Taggart, J. V. (1953). J. biol. Chem. 203, 925.

Schachter, D. \& Taggart, J. V. (1954). J. biol. Chem. 208, 263.

Stadtman, E. R. \& Kornberg, A. (1953). J. biol. Chem. 203, 47.

Stadtman, E. R., Novelli, G. D. \& Lipmann, F. (1951). J. biol. Chem. 191, 365.

\title{
A Ghromatographic Method for the Estimation of Nicotinuric Acid and Nicotinamide
}

\author{
BY K. M. JONES \\ Department of Biochemistry, University of Oxford
}

\section{(Received 14 April 1959)}

The published methods for the estimation of nicotinuric acid in mixtures containing nicotinic acid and other related compounds depend either upon the colorimetric estimation of 'total nicotinic acid' before and after hydrolysis, different methods of hydrolysis being employed to convert nicotinuric acid and other derivatives into nicotinic acid (for example, Ellinger \& Abdel Kader, 1949), or upon the microbiological assay of nicotinic acid with two micro-organisms, one of which also responds to nicotinuric acid whereas the other does not (Johnson, 1945). Since they measure nicotinuric acid by difference, these methods are not able accurately to estimate small amounts of this substance in the presence of a large excess of nicotinic acid, and consequently were unsuitable for use in the experiments described in the two previous papers (Jones \& Elliott, 1959; Jones, 1959).

This paper describes a direct method for nicotinuric acid estimation, in which nicotinuric acid is first separated from interfering substances by paper chromatography and then estimated by measurement of its u.v. absorption after elution from the paper. This method has also been successfully adapted to the estimation of nicotinamide in the presence of nicotinic acid.

\section{EXPERIMENTAL}

Materials and general methods have been described by Jones \& Elliott (1959).
Estimation of nicotinuric acid. Samples containing 5$100 \mu \mathrm{g}$. of nicotinuric acid were spotted on to Whatman no. 3 paper to produce spots of $1 \mathrm{~cm}$. diameter. The chromatograms were then developed in descending butan-1-ol saturated with water, for $18-48 \mathrm{hr}$., the solvent being allowed to drip off the bottom of the papers. After solvent development, chromatograms were dried for $30 \mathrm{~min}$. at 50$60^{\circ}$. The spots of nicotinuric acid were located by making a contact print of the chromatogram by the method of Markham \& Smith (1949) with unfiltered light from a mercury lamp. By making a tracing of the photograph and transferring this on to the chromatogram, the positions of the spots could be fixed exactly.

The spots of nicotinuric acid were cut out, each with a border the size of which was then adjusted so that all the pieces were of equal weight. It was generally possible to include the whole of the nicotinuric acid spot in $200 \mathrm{mg}$. of paper (about $10 \mathrm{~cm} .{ }^{2}$ ). The pieces of paper were cut into strips 1-2 mm. wide and put in test tubes. A volume (5 ml.) of $\mathrm{N}-\mathrm{HCl}$ was then added to each tube and they were left to stand with occasional shaking for $3 \mathrm{hr}$., in which period elution was found to be complete. After $3 \mathrm{hr}$. the fluid was poured off, centrifuged to remove small shreds of paper and its extinction was measured at $262 \mathrm{~m} \mu$ against $\mathrm{N}-\mathrm{HCl}$. Simultaneously a piece of blank paper was cut from the band having the same $R_{F}$ as nicotinuric acid and eluted exactly as described above. The extinction of this eluate constituted the 'paper blank' which was deducted from the experimental values. The amount of nicotinuric acid was calculated from a calibration curve separately constructed as described below.

Estimation of nicotinamide. Nicotinamide was estimated by the method described above for nicotinuric acid estimation except that chromatograms were developed for only 